\title{
Evaluación de desempeño en concretos adicionados con microsílica
}

\begin{tabular}{l|l} 
C. Martínez & E. Vera \\
\end{tabular}

Recibido:

Julio 23 de 2009

Aceptado:

Mayo 19 de 2010
1. Universidad Pedagógica y

Tecnológica de Colombia,

Carretera Central del Norte

- Tunja, Colombia

Grupo de Superficies

Electroquímica y Corrosión

(GSEC)

eduardo.martinez@uptc.edu.co, enrique.vera@uptc.edu.co.

\section{Resumen}

El presente artículo muestra los resultados sobre el estudio del efecto de la adición microsílica (Síkafume) al cemento (portland tipo I), con el fin de mejorar las propiedades mecánicas y anticorrosivas del concreto. Dos mezclas ( $m 1$ y m2) fueron fabricadas con igual relación Agua Cemento(A/C). Ensayos de resistencia mecánica, granulometría y ensayo de succión capilar se llevaron a cabo para caracterizar las propiedades estructurales del concreto. Para evaluar las propiedades anticorrosivas, se llevaron a cabo pruebas de espectroscopia de impedancia electroquímica (EIS) y resistencia a la polarización lineal (LPR). Los resultados muestran que la adición de microsílica mejora las propiedades tanto mecánicas como anticorrosivas del respectivo concreto.

Palabras clave: Microsílica, resistencia a la compresión, corrosión, electroquímica.

\section{Abstract}

The purpose of this article is to show the results of a study about effects of adding microsilica (Sikafume) to cement (Portland type I) in order to improve the mechanical corrosion properties of concrete. Two mixtures were elaborated ( $\mathrm{m} 1$ y $\mathrm{m2}$ ) with the same amount of water and cement. Different tests were carried out in order to characterize the structural properties of concrete: mechanical resistance, granulometry and capillary suction test. Electrochemical Impedance Spectroscopy (EIS) and Linear Polarization Resistance (LPR) techniques were used to evaluate the anti-corrosion properties. The results show that adding microsilica improves both the mechanical and corrosion properties of the respective concrete.

Key Words: microsilica, strength compression, corrosion, electrochemical. 


\section{Introducción}

$\mathrm{L}$ os concretos por su naturaleza de ser obtenidos por la unión de diferentes agregados, deben tener un procedimiento y un control adecuado para lograr unos buenos resultados [1]. Aunque esto no es un factor decisivo a la hora de usarlo, si es conveniente cuando se desean alcanzar unos resultados óptimos en una obra.

Evidentemente factores de seguridad en el diseño engloban todos los posibles fallos que pueden ocurrir. "Cuando cambia las condiciones de servicio de la estructura el concreto se altera o a través de él penetran sustancias agresivas, que desencadenan en un proceso corrosivo con una triple consecuencia: a) el acero disminuye su sección o incluso se convierte completamente en oxido; b) debido a las presiones que ejerce el oxido al generarse, el concreto puede fisurarse o desintegrarse; c) la adherencia acero-concreto disminuye o desaparece" [2]. Adicionalmente procesos de carbonatación se suman para disminuir el pH del concreto y favorecer la ruptura de la capa pasiva del acero [3].

Para proteger de la corrosión los aceros embebidos en el concreto se suele adicionar al concreto microsílica, producto que es más fino que el cemento y que tiene dos formas de reaccionar: 1) una reacción puzolánica y 2) un efecto de llenado [4].

En cuanto a las propiedades del concreto la adición de puzolana, protege la armadura de acero, pues disminuye los poros en la matriz de concreto, evitando así la entrada rápida de cloruros que puedan romper la capa pasiva del acero. La adición de microsílica en la matriz del concreto disminuye la permeabilidad [5].

Las técnicas para valorar la velocidad de corrosión (VC) son muy variadas entre las que se destacan la LPR y la EIS. Las anteriores técnicas ofrecen ventajas frente a otras respecto a la cantidad de datos que aportan y la eficacia de sus resultados, ventajas que se reflejan en un buen valor en la determinación de la velocidad de corrosión y así tomar decisiones acertadas en caso de proteger una estructura.

\section{Montaje experimental}

Dos mezclas $\mathrm{m} 1, \mathrm{~m} 2$ se fabricaron utilizando cemento portland tipo I, con la misma proporción agua/cemento (tabla No. 1). Una varilla ASTM-A706 [6] de $1.27 \mathrm{~cm}$ de diámetro $(\varnothing=1 / 2$ pulgada) y $11 \mathrm{~cm}$ de longitud, se utilizó para simular el acero de refuerzo, a la que se le aplicó una película aislante, para dejar un área activa de 31.92 $\mathrm{cm} 2$, según se muestra en la fig. 1 .

Tabla No. 1 Proporciones de mezclas utilizados para los ensayos

\begin{tabular}{|c|c|c|}
\hline Elemento $\quad$ Mezcla & $\mathrm{m} 1$ & $\mathrm{~m} 2$ \\
\hline Agua/Cemento (W/C) & 0.6 & 0.6 \\
\hline Cemento+Adición Puzolánica (C+AP*) & $0.9+0.1$ & $1+0$ \\
\hline Arena (A) & 2 & 2 \\
\hline Gravilla (G) & 2 & 2 \\
\hline
\end{tabular}

*AP=Adición Puzolánica

A la mezcla $\mathrm{m} 1$ se reemplazó el 10\% del total en peso del cemento por una adición de puzolana (AP) para concretos de alta resistencia Sikafume (a base de microsílica). Las cantidades de arena (A) y de gravilla $(G)$ se indican en la tabla No.1. El curado de las probetas inició después de 24 horas de secado, del cual se desencofraron y se sumergieron en la piscina de curado, en donde permanecieron durante 7 días. 
Fig. 1 Montaje de las probetas para los ensayos electroquímicos

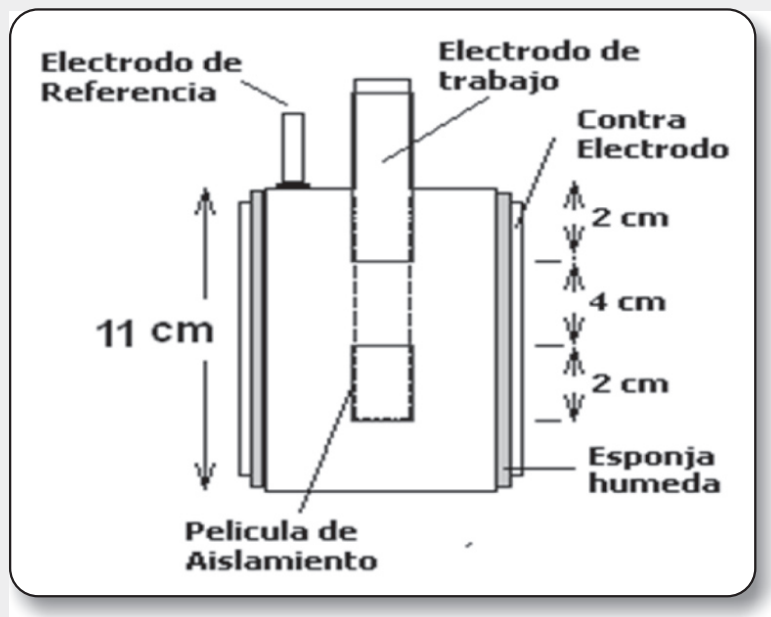

Las probetas se sumergieron en un ambiente corrosivo, compuesto por agua destilada al 3.5\% de $\mathrm{NaCl}$. La evaluación de los parámetros electroquímicos se llevo a cabo usando la técnica de Resistencia a la Polarización Lineal (ASTM G59[7])y la técnica de Espectroscopia de Impedancia electroquímica (EIS; ASTM G106 [8]). Se utilizó un equipo potenciostato-galvanostato GAMRY PCI $14 / 750^{\mathrm{TM}}$ que utiliza como soporte la aplicación EIS 300. Se utilizó como referencia en todos los ensayos un electrodo de plata cloruro de plata $(\mathrm{Ag} / \mathrm{AgCl})$.

\section{Discusión de los resultados}

\subsection{ANÁLISIS GRANULOMÉTRICO AGREGADO FINO (ARENAS)}

Para la caracterización de los materiales, se realizaron ensayos de granulometría a partir del tamizado de muestras, de los agregados finos y gruesos, los resultados se muestran en las figuras 2 y 3 . Los tamices se ajustaron según los criterios establecidos en la norma NTC-32[9] y las operaciones de tamizado se llevaron a cabo según la norma NTC-77[10].
Fig. 2 Análisis Granulométrico del agregado fino

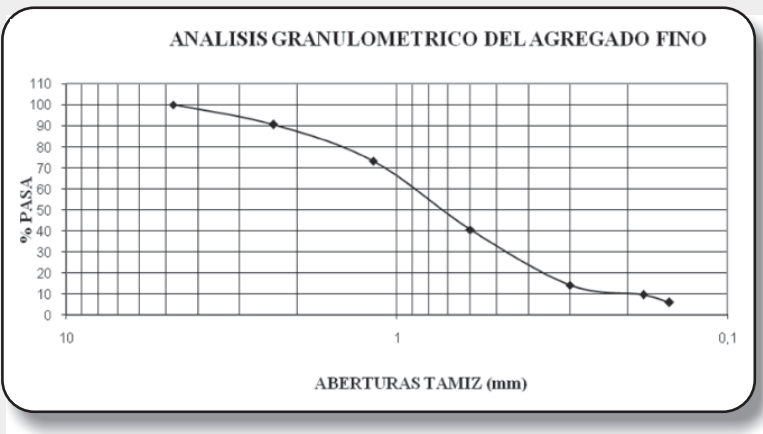

La indicación de la gradación puede determinarse numéricamente de la curva de tamaño de los granos, para la fracción mayor de la malla No 200, usando el coeficiente de uniformidad $\left(\mathrm{Cu}=\mathrm{D}_{60} / \mathrm{D}_{10}\right)$ y por el coeficiente de concavidad $\left(\mathrm{Cc}=\left[\mathrm{D}_{30}\right]^{2} /\left[\mathrm{D}_{10}\right.\right.$ D60]). Un valor alto de $\mathrm{Cu}$ indica una gama amplia de tamaños de los granos, entre D60 y $\mathrm{D}_{10}$ (tamaño de grano del $60 \%$ que pasa y tamaño del $10 \%$ que pasa).

Tabla No. 2 Determinación de Cu y Cc para agregado fino.

\begin{tabular}{|c|c|c|c|c|}
\hline $\mathbf{D}_{\mathbf{6 0}}$ & $\mathbf{D}_{\mathbf{3 0}}$ & $\mathbf{D}_{\mathbf{1 0}}$ & $\mathbf{C u}=\mathbf{D}_{\mathbf{6 0}} / \mathbf{D}_{\mathbf{1 0}}$ & $\mathbf{C c}=\left(\mathbf{D}_{\mathbf{3 0}}\right)^{2} /\left(\mathbf{D}_{\mathbf{1 0}} \mathbf{D}_{\mathbf{6 0}}\right)$ \\
\hline 0.9 & 0.45 & 0.21 & $\mathbf{4 . 3}$ & $\mathbf{1 . 0 7}$ \\
\hline
\end{tabular}

Un valor de Cc de aproximadamente 1.00 indica una variación altamente lineal de la curva granulométrica, entre D60 y D10 cuando $\mathrm{Cu}$ tiene un valor de 4 y 6 . El valor de Cu de 4.3 indica la existencia de un tamaño de grano aproximadamente 4 veces mayor de 0.21 $\mathrm{mm}$. El valor de los porcentajes se encuentra relacionado en la tabla No. 2, según los valores obtenidos de la curva granulométrica de la fig. 2. La gradación del agregado fino (Arena) se utilizó en cada una de las mezclas (m1 y $\mathrm{m} 2)$ en las proporciones indicadas (Ver tabla No. 1).

\subsection{ANÁLISIS GRANULOMÉTRICO AGREGADO GRUESO (GRAVILLA)}

El valor de los diámetros $(\mathrm{mm})$ se incluyen en la tabla No. 3. Según lo observado en la curva granulométrica para el agregado grueso de la figura 3. 

para la gravilla

\begin{tabular}{|c|c|c|c|c|}
\hline $\mathrm{D}_{60}$ & $\mathrm{D}_{30}$ & $\mathrm{D}_{10}$ & $\mathrm{Cu}=\mathrm{D}_{60} / \mathrm{D}_{\mathbf{1 0}}$ & $\mathrm{Cc}=\left(\mathrm{D}_{30}\right)^{2} /\left(\mathrm{D}_{\mathbf{1 0}} \mathrm{D}_{60}\right)$ \\
\hline 21 & 17 & 15 & 1.4 & 0.92 \\
\hline
\end{tabular}

La gravilla está bien gradada ya que su coeficiente de concavidad, tiene un valor de $\mathrm{Cc}=0.92$. El valor de $\mathrm{Cu}$ de 1.4 indica una variación en el tamaño de la gravilla de 1.5 veces respecto al tamaño que paso el $10 \%$.

Fig. 3 Análisis Granulométrico del agregado grueso

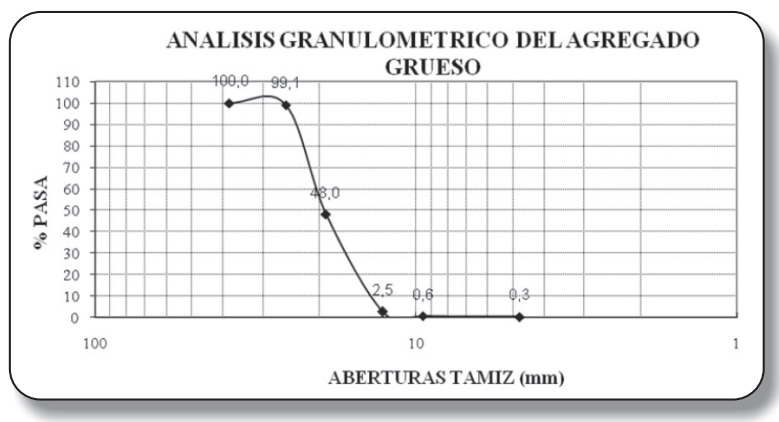

La gradación del agregado grueso (Gravilla) se utilizó en cada una de las mezclas ( $\mathrm{m} 1$ y $\mathrm{m} 2$ ) en las proporciones indicadas (Ver tabla No. 1).

\subsection{ANÁLISIS DEL ENSAYO DE} RESISTENCIA A LA COMPRESIÓN AXIAL

Con este ensayo se determinó el esfuerzo a compresión de las muestras cilíndricas de concreto fabricadas con moldes específicamente para esta investigación a los 7 y 28 días de curado. El procedimiento lo describe la norma ASTM C39[11].

Para las mezclas $\mathrm{m} 1$ y $\mathrm{m} 2$ se verificaron sus resistencias a la compresión con cilindros de concreto. Se construyeron moldes de PVC $(\varnothing=3 ")$ de $7.62 \mathrm{~cm}$ de diámetro y una altura de (6") $15.24 \mathrm{~cm}$, con una abertura lateral para permitir desencofrar la probeta, con una relación $\mathrm{h} / \varnothing=2$ (altura/diámetro $=2$ ), para no realizar correcciones. Se fallaron en una máquina universal Shimadzu UH-500 KNl.
En la tabla No. 4 se evidencia que aumentó un $33.7 \%$ la resistencia promedio de la mezcla a la que se le adicionó microsílica, notoriamente mayor que la obtenida para la mezcla $\mathrm{m} 2$, con un porcentaje de 8.5 a los 28 días.

Tabla No. 4 Porcentaje de aumento en las resistencias mecánicas a los 7 y 28 días de curado

\begin{tabular}{|c|c|c|}
\hline Curado Mezcla & $\begin{array}{c}\mathbf{m} \mathbf{1} \\
\left(\mathbf{N} / \mathbf{m}^{2}\right)\end{array}$ & $\begin{array}{c}\mathbf{m} \mathbf{2} \\
\left(\mathbf{N} / \mathbf{m}^{\mathbf{2}}\right)\end{array}$ \\
\hline 7 días & 8253351 & 8906251 \\
\hline 28 días & 11032808 & 9664334 \\
\hline \% Aumento & 33.7 & 8.5 \\
\hline
\end{tabular}

En la figura 4 se muestra gráficamente los resultados obtenidos de la resistencia a la compresión.

Fig. 4 Resultados de las resistencias promedio a la compresión axial para las mezclas $\mathrm{m} 1$ y $\mathrm{m} 2$ a los 7 y 28 días de curado.

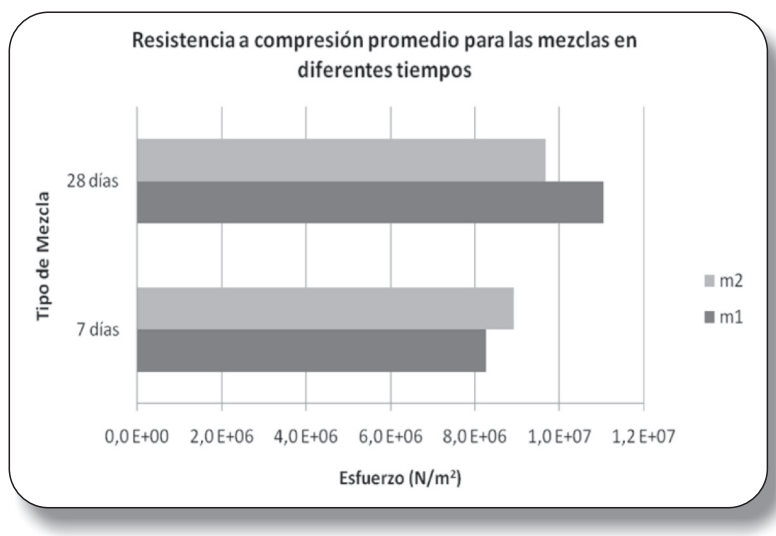

La microsílica reacciona químicamente con el hidróxido de calcio $(\mathrm{CH})$ para producir una cantidad adicional de silicato de calcio hidratado (C-S-H), el cual aumentó la resistencia del concreto. Hecho que no se evidencio en las demás mezclas que carecían de esta adición puzolánica.

\subsection{ENSAYO DE SUCCIÓN CAPILAR EN CONCRETOS DE CEMENTO HIDRÁULICO}

Con este ensayo se determinó la relación de absorción de agua mediante mediciones en el incremento en la masa de la muestra, producto de la absorción de agua como función del tiempo cuando una sola superficie de la muestra 
está expuesta al agua, el procedimiento lo describe la SIA 162/1 o la ASTM C1585[12]. En esta técnica se determinan parámetros como: El coeficiente de absorción capilar (K), la resistencia a la penetración de agua $(\mathrm{m})$ y el tiempo de saturación de la probeta $(\mathrm{T})$.

En la figura 5 se observa que las probetas con microsílica presentaron un mejor comportamiento a la succión capilar (debido a las buenas propiedades que le da la adición), ya que evidenciaron menor tiempo de saturación, comparado con el tiempo obtenido por la mezcla sin adición. Estos resultados se asocian con el tamaño de poro que integraban la mezcla de concreto, siendo más pequeños en la mezcla con microsílica, y de mayor tamaño en la mezcla carente de esta.

Fig. 5 Resultados promedio de los ensayos de succión capilar para las mezclas $\mathrm{m} 1 \mathrm{y} \mathrm{m} 2$.

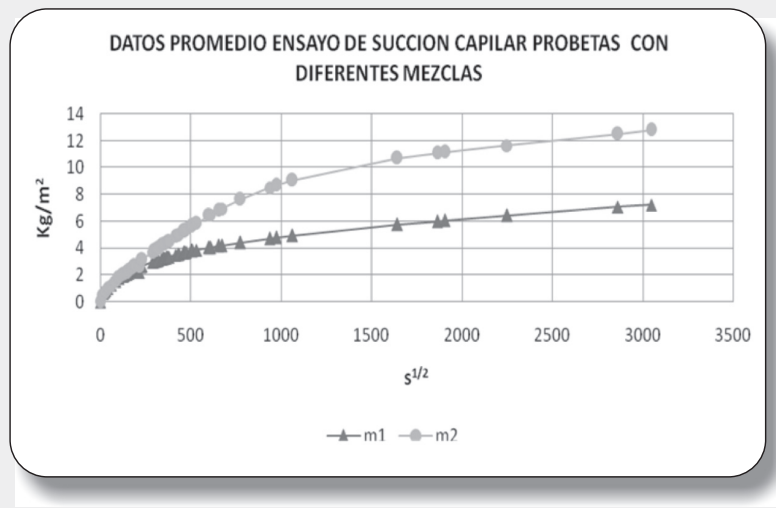

En la tabla No. 5 se observan los valores calculados para las gráficas de la figura 5 , se ve que el valor de coeficiente de succión capilar para las probetas adicionadas con microsílica es más pequeño respecto de las probetas sin adición. Ésta pendiente es solo un indicativo para la primera porción de la curva, que se encuentra en el rango de 0 a $250 \mathrm{~s}^{1 / 2}$; en el rango de 1500 a $3000 \mathrm{~s}^{1 / 2}$ se determina otra recta que corta a la primera en un punto, de tal forma que a cada mezcla se calcula este intercepto. Este punto indica el tiempo de saturación $(\mathrm{T})$, de esta forma el valor de $\mathrm{T}$ para la mezcla sin adición es mayor comparado con el $\mathrm{T}$ que necesito la mezcla adicionada con microsílica.
Tabla No. 5 Coeficiente K, tiempo de saturación (T), altura de la probeta $(Z)$ y resistencia a la penetración $(\mathrm{m})$

\begin{tabular}{|c|c|c|c|c|c|}
\hline Mezcla & $\begin{array}{c}\mathrm{K} \\
\left(\mathrm{kg} / \mathrm{m}^{2} \mathrm{~s}^{12}\right)\end{array}$ & $\mathrm{T}(\mathrm{s})$ & $\mathrm{Z}(\mathbf{m})$ & $\mathrm{Z}^{2}\left(\mathrm{~m}^{2}\right)$ & $\mathbf{m}=\mathrm{T} / \mathrm{Z}^{2}$ \\
\hline $\mathrm{ml}$ & 0.0011 & 132060 & 0.1087 & 0.01181 & $1.12 \mathrm{E} 07$ \\
\hline $\mathrm{m} 2$ & 0.0013 & 451136 & 0.1053 & 0.01109 & $4.07 \mathrm{E} 07$ \\
\hline
\end{tabular}

En la tabla No 5 se muestra que la resistencia a la penetración $(\mathrm{m})$ es mucho mayor (en alrededor de un 264\%) para la mezcla sin adición que para la probetas adicionadas con microsílica.

\subsection{ENSAYOS ELECTROQUÍMICOS}

Se usaron dos técnicas $[13,14,15,16]$ para la determinación de la velocidad de corrosión (VC) del acero embebido en el concreto, la primera de ellas es más comúnmente aplicada en campo, la LPR, en tanto que la técnica EIS, tiene la ventaja de proporcionar mayor información. La mezcla $\mathrm{m} 1$ y $\mathrm{m} 2$ tienen la misma relación $\mathrm{A} / \mathrm{C}=0.6$, sumergiéndose las probetas en una solución salina $(3.5 \% \mathrm{NaCl})$.

Resistencia a la polarización lineal (LPR): $\mathrm{Al}$ contener iones en la solución $(3.5 \% \mathrm{NaCl})$, las lecturas en las medidas evidenciaron que la mezcla adicionada con microsílica tuvo un mejor comportamiento al fenómeno de la corrosión en los primeros 60 días (fig. 6), ya que sus valores de $\mathrm{Rp}$ superaron los valores obtenidos para la mezcla sin adición, y se nota un ligero aumento de los valores a partir de los 90 días.

Fig. $6 \mathrm{Rp}$ (promedio) para las mezclas $\mathrm{m} 1 \mathrm{y} \mathrm{m} 2$ sumergidas en agua destilada $(3.5 \% \mathrm{NaCl})$ según el tiempo de exposición.

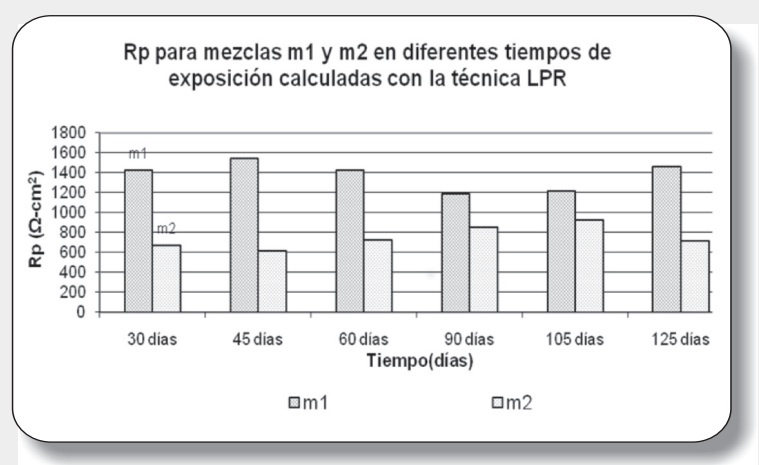


Espectroscopia de Impedancia Electroquímica (EIS): En la fig. 7 se observan los valores de Rp para las probetas sumergidas en $3.5 \% \mathrm{NaCl}$, donde nuevamente los valores de la mezcla adicionada con microsílica superaron a los valores de la mezcla sin adición a lo largo del tiempo.

Fig. $7 \mathrm{Rp}$ (promedio) para las mezclas $\mathrm{m} 1 \mathrm{y} \mathrm{m} 2$ sumergidas en agua destilada $(3.5 \% \mathrm{NaCl})$ según el tiempo de exposición.

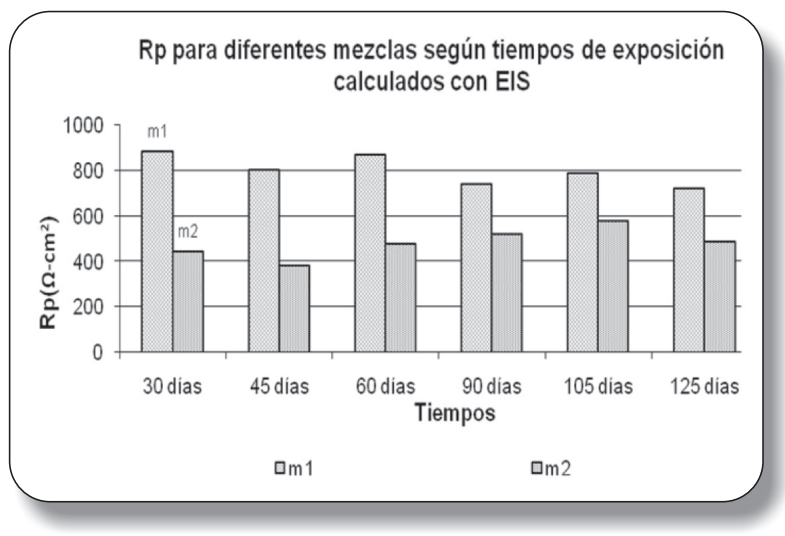

Sin embargo se ve aumento del valor $\mathrm{Rp}$ de la mezcla sin microsílica en los últimos tiempos, posiblemente por el crecimiento acelerado de los productos de hidratación del cemento (óxidos, hidróxidos y oxihidróxidos) de la interfase.

\section{VELOCIDAD DE CORROSIÓN} OBTENIDAS CON LAS TÉCNICAS LPR Y EIS

En la fig. 8 se observan las VC del acero embebido en las dos mezclas.
Fig. 8 Velocidad de corrosión ( $\mathrm{mm} / \mathrm{y})$ obtenidas de las mezclas $\mathrm{m} 1$ y $\mathrm{m} 2$ sumergidas en agua destilada $(3.5 \%$ $\mathrm{NaCl}$ ) según el tiempo de exposición

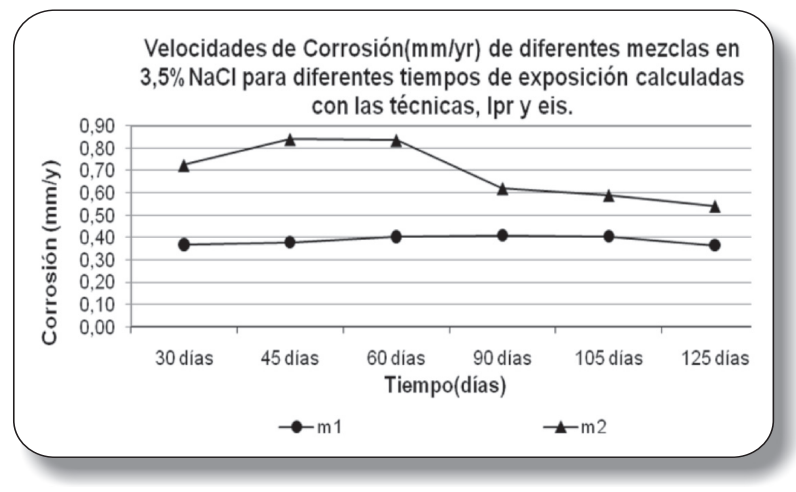

En la gráfica 8 se ve la variación de las VC de la mezcla sin adición, respecto de la mezcla con microsílica. Las mezclas con microsílica mostraron velocidades de corrosión en el rango de los 0.37 a los $0.31 \mathrm{~mm} / \mathrm{y}$, mucho más pequeño que el rango para la mezcla sin adición, que estuvo entre 0.83 a $0.51 \mathrm{~mm} / \mathrm{y}$.

\section{Conclusiones}

Las probetas con adición de microsílica, aumentaron en un $33.7 \%$ la resistencia a la compresión axial, de los 7 a los 28 días. Porcentaje significativo respecto a la mezcla no adicionada, ya que tenían la misma relación $\mathrm{A} / \mathrm{C}$.

La succión capilar para la mezcla con microsílica disminuyó su tiempo de saturación, conllevando a una mejoría en sus propiedades tan solo con un cambio en el cemento del $10 \%$ en peso.

La protección al ingreso de agua es mayor en la mezcla a la que se le adicionó la microsílica, teniendo un valor de resistencia a la penetración de 1.12 , menor respecto de la mezcla que duraron 2.4 veces más, que corresponde a la mezcla sin adición.

Los valores obtenidos de $\mathrm{VC}$ de las mezclas adicionadas con microsílica demuestran una disminución en la velocidad de corrosión, respecto de la mezcla carente de esta. El rango de variación para las lecturas fue más estable con la mezcla adicionada con microsílica respecto de la mezcla sin adición.

\section{Agradecimientos}

Los autores expresan agradecimientos a la UPTC, por el apoyo financiero para realizar el proyecto y a Sika por facilitarnos la adición puzolánica para los ensayos. 


\section{Bibliografía}

[1] SÁNCHEZ DE GUZMÁN, D. (2001). Tecnología del concreto y del mortero. Bhandar Editores. 349 p.

[2] OLGUIN, F. ALMERAYA, F. OROZCO, V. BORUNDA, A. GAONA, C. MARTÍNEZ, A. TORRES, A. (2004). Influence of environmental and electrochemical factors in the durability of concrete. Portugaliae electrochimica acta 22. p. 321-334.

[3] DAILY, Steven F. (1999). Understanding Corrosion and Cathodic Protection of Reinforced Concrete Structures. Corrpro Companies : Technical Paper Library, CP-48. 5 p.

[4] GIANNOTTI, F. \& LIBARDI, J. (2006) A study of steel bar reinforcement corrosion in concretes with SF and SRH using Electrochemical Impedance Spectroscopy. Materials Research, Vol. 9, No. 2. p. 209-215.

[5] JOUKOSKI, A., PORTELlA, K. Baron, O. et. al. (2004) The influence of cement type and admixture on life span of reinforced concrete utility poles subjected to the high salinity environment of Northeastern Brazil, studied by corrosion potential testing. Cerâmica 50. p. 12-20.

[6] ASTM-A706. Standard Specification for Low-Alloy Steel Deformed and Plain Bars for Concrete Reinforcement. 6p.

[7] ASTM-G059. Test Method for ConductingPotentiodynamicPolarization Resistance Measurements. 4p.

[8] ASTM-G106. Practice for Verification of Algorithm and Equipment for Electrochemical Impedance Measurements. 11p.
[9] NTC-32. Tejido de alambre y tamices para propósitos de ensayo. 11p.

[10] NTC-77. Método para el Análisis por Tamizado de los Agregados Finos y Grueso.

[11] ASTM-C39. Test Method for Compressive Strength of Cylindrical Concrete Specimens. 5p.

[12] ASTM C1585. Test Method for Measurement of Rate of Absorption of Water by Hydraulic-Cement Concretes. $6 \mathrm{p}$.

[13] BENTUR, A., DIAMOND, S. \& BERKE, N. (1997). Steel Corrosion in Concrete (Fundamentals and civil engineering practice). Great Britain. Editorial Chapman y Hall. 220 p.

[14] MONTEMOR, M. SIMOES, A. FERREIRA, M. (2003). Chlorireinduced corrosion on reforcing steel: from the fundamental to the monitoring techniques. Cement \& Concrete Composites 25. p. 491-502.

[15] ISMAIL, M \& OHTSU, M. (2006). Corrosion rate of ordinary and highperformance concrete subjected to chloride attack by AC impedance spectroscopy. Construction and Building Materials 20. p. 458-469

[16] NATIONAL READY MIXED CONCRETE ASSOCIATION. ¿Qué, por qué y cómo? Corrosión del Acero en el Concreto : El Concreto en la Práctica(CIPes 25). Estados Unidos: NRMCA, 2p. 1998. 